\title{
ALICE: Angled Label Image Capture and Extraction for high throughput insect specimen digitisation
}

Ben Price $^{1 *}$, Steen Dupont ${ }^{1}$, Elizabeth Louise Allan ${ }^{1}$, Vladimir Blagoderov ${ }^{1,2}$, Alice Butcher ${ }^{1}$, James Durrant $^{1}$, Pieter Holtzhausen ${ }^{3}$, Phaedra Kokkini ${ }^{1}$, Laurence Livermore ${ }^{1}$, Helen Hardy ${ }^{1}$ and Vince Smith ${ }^{1}$

${ }^{1}$ Natural History Museum, Cromwell Road, SW7 5BD, London

${ }^{2}$ National Museums Scotland, Chambers Street, EH1 1JF, Edinburgh

${ }^{3}$ Centerforest, Oranjelaan 7, Stellenbosch, 7600, South Africa

*Corresponding author: b.price@nhm.ac.uk

\section{Abstract}

The world's natural history collections contain at least 2 billion specimens, representing a unique data source for answering fundamental scientific questions about ecological, evolutionary, and geological processes. Unlocking this treasure trove of data, stored in thousands of museum drawers and cabinets, is crucial to help map a sustainable future for ourselves and the natural systems on which we depend. The rate-limiting steps in the digitisation of natural history collections often involve specimen handling due to their fragile nature. Insects comprise the single largest collection type in the Natural History Museum, London (NHM), reflecting their global diversity. The NHM pinned insect collection, estimated at 25 million specimens, will take over 700 person years to digitise at current rates. In order to ramp up digitisation we have developed ALICE for Angled Label Image Capture and Extraction. This multi-camera setup and associated software processing pipeline enables primary data capture from angled images, without removal of the labels from the specimen pin. As a result ALICE enables a single user to sustainably image over 1,000 specimens per day, allowing us to digitally unlock the insect collections at an unprecedented rate.

\section{Introduction}

The world's natural history collections contain at least 2 billion specimens, of which an estimated $55 \%$ reside in Europe (Ariño, 2010). Collected over 500 years of human exploration and still constantly expanding, these specimens are a vast and underused repository of information on the natural world, allowing us to explore two great time scales: first, the dynamic geological history of our planet and its life spanning 4.56 billion years, and second, the impact of humans on that natural world over the last few thousand years. Natural history collections represent a unique data source and research tool for meeting the most important challenge humans face over the next 30 years - mapping a sustainable future for ourselves and the natural systems on which we depend - and for answering fundamental scientific questions about ecological, evolutionary, and geological processes and how they interact to shape our changing planet (Suarez \& Tsutsui, 2004).

Recent advances in digital and genomic technologies promise to transform the scientific impact of natural history collections, with huge new potential for addressing societal challenges ranging from biodiversity loss and food security to climate change and neglected and emerging tropical diseases. The Earth BioGenome Project (Lewin et al., 2018) has recently been proposed to address the genomic component of this challenge. By sequencing, cataloging, and characterising the genomes of all of Earth's eukaryotic biodiversity over a period of 10 years, the group make the case for farreaching benefits of creating an open digital repository of genomic information for life on Earth. Fully realising the benefits of such a project to address challenges such as the impact of climate change on 
biodiversity, the conservation of endangered species and ecosystems, and the preservation and enhancement of ecosystem services, requires far more information than just the genomic data of these species. Resynthesising biology in the way Lewin et al. (2018) suggest, requires us to understand the organism in its environment, reconnect it to its evolutionary past; and model the complexity of the organism, its evolution, and the environment. Unlocking this potential, requires in part, the connection of this vast genomic archive with the treasure trove of data and information currently stored in thousands of museum drawers and cabinets, and in the vast repositories of natural science publications, to make this universally available for maximal scientific potential.

Within the Natural History Museum, London (NHM) the Digital Collections Programme aims to freely and openly release data by digitising the estimated 80 million specimens in the collection, to increase access to, and use of the collections by researchers and others, and to allow that data to form part of a research infrastructure across multiple collections, which can be used at scale alongside other data sources such as climate or population data.

Insects comprise the largest collection within the NHM, with 25 million pinned specimens of the estimated 80 million total, thus pinned insects likely account for the antlion's share of the global two billion specimen total. At present only 1.3 million insect specimens ( $4 \%$ of the NHM collection) have digital records available online through the NHM Data Portal http://data.nhm.ac.uk/; even with this small percentage available, we are seeing exceptional promise in downloads and citations - but most of the NHM pinned insect collections remain largely unknown to the majority of potential users, with access limited to visits in person or specimen loans.

Digitisation of insects currently takes three primary approaches: (1) data capture through direct transcription, without imaging; (2) whole drawer scanning (e.g: Blagoderov et al., 2012; Dietrich et al., 2012; Hudson et al., 2015); and (3) single specimen imaging with label capture (e.g: Paterson et al., 2016; Blagoderov et al., 2017), with or without immediate transcription. Direct transcription is ratelimited by physical access to the collection, and crucially data cannot be checked without examining the specimen labels again. Whole drawer scans provide a quick overview of the collection and can provide a specimen inventory of the collection, but do not provide additional label data due to the overlap of the specimen in the image.

Current single specimen label imaging methods require removal of the labels from the pinned specimen (eg. the iCollections project: Paterson et al., 2016; Blagoderov et al., 2017). This specimen preparation can comprise $\sim 50 \%$ of the time taken to digitise each specimen (Blagoderov et al., 2017), increases the specimen handling and thus the risk of specimen damage. Additional methods involve the use of conveyor belts with 3D printed specimen stages and two cameras: one above the specimen and one at an angle (Tegelberg et al., 2014), or more recently the use of an array of light field cameras including 3D capture (Hereld et al., 2017).

As specimen digitisation involves several steps it is difficult to compare published estimates meaningfully as they include different setups, workflows, numbers of operators and levels of collection preparation and data transcription. With this caveat in mind the currently published rates with minor specimen preparation and data transcription are 41 - 62 specimens per person per hour (Tegelberg et al., 2017) or 250 specimens per person per day (Tegelberg et al., 2014).

Within the NHM the iCollections project digitised 380,686 British and Irish Lepidoptera and 33,365 Diptera, removing labels before imaging (Blagoderov et al. 2017). The iCollections base rate $(E)$ for preparation and imaging was 24 - 32 specimens per person per hour (recalculated from Table 6 : Blagoderov et al., 2017) depending on the collection, with the digitisation of the Diptera collection being slower than the Lepidoptera collection. The entire end to end process of preparation, imaging 
and transcription, without site georeferencing, was estimated at 15 - 25 specimens per person per hour (recalculated from Table 6: Blagoderov et al., 2017). At these published rates, the digitisation of the NHM insect collection will take over seven hundred person years to complete.

The rate-limiting steps in the digitisation of natural history collections involve specimen handling due to the precious and fragile nature of the material. Robotic handling of pinned insects (e.g. Nguyen et al., 2017) may increase the daily handling rate, however this is unlikely to reach the rate of image capture without multiple robotic platforms feeding a single imaging station. In order to reduce the specimen handling bottleneck we examined different approaches to imaging the labels at an angle while they remain in place on the pin, with the primary focus being the top label, and with occasional manual separation of the top label if pressed against the specimen itself. The solution here presented is Angled Label Image Capture and Extraction, or ALICE.

\section{Design and Specification}

It was clear from initial testing that while angled images were feasible, multiple views were required to overcome the pin and specimen blocking information on the top label, and the labels blocking each other. To provide these multiple views, two hardware approaches were tested: (1) rotating the specimen stage; and (2) multiple cameras imaging in parallel. To facilitate simple and cost effective re-implementation of these solutions in other collections, consumer cameras were selected over scientific cameras due to their lower cost, ease of use and the ability to use "off the shelf" software for tethered camera control.

\section{Rotating stage with single camera}

Initial tests used a single DSLR setup (either the Nikon D5300 with a Nikon $40 \mathrm{~mm}$ macro lens; or Canon 5DS $R$ with a Canon $100 \mathrm{~mm}$ macro lens) mounted on a copy stand at approximately 35 degrees off horizontal, with a flash for lighting, and either (1) a manually rotated, repurposed microscope slide turntable, or (2) the Cognisys rotary table (https://www.cognisysinc.com/store/rotary-table.html) controlled with the Stackshot 3X controller (https://www.cognisysinc.com/products/stackshot3x/stackshot3x.php) and Helicon Remote v.3.9.7

(https://www.heliconsoft.com/heliconsoft-products/helicon-remote/). For each specimen four to six images were taken, rotating the stage by $60^{\circ}$ to $90^{\circ}$ between images (Figure 1). Specimen rotation using the Stackshot controller proved to be a rate-limiting step, and manual rotation was not a sustainable solution for daily implementation on a large scale project, thus multiple cameras imaging in parallel was prefered. However, as the single camera solution is more portable and amenable to smaller projects we include the specifications for potential use in smaller collections or by researchers visiting collections for a short period of time. 


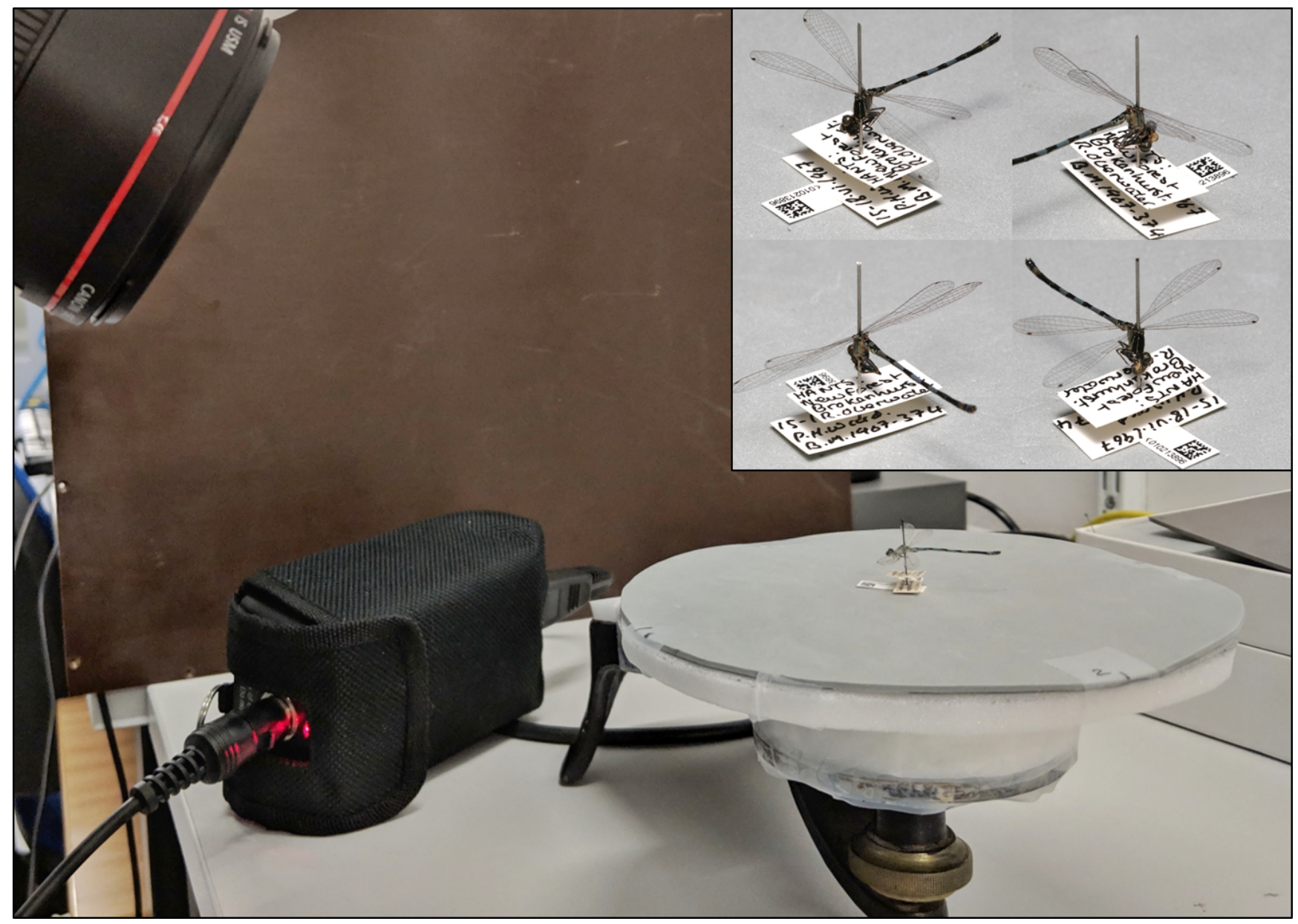

Figure 1: Rotational stage, a repurposed microscope slide turntable, with resulting label images inset. Note: label images are cropped for display.

\section{Multiple cameras imaging in parallel}

Initial tests confirmed that at least two images from different viewpoints were required to provide an unobstructed view of the entire top label. However, in order to to maximise the image focal plane over the labels at feasible apertures we designed the setup with four angled cameras focussed on the top label and an additional two cameras to provide basic lateral and dorsal overview specimen images (Figure 2). The four label imaging cameras and lateral camera are mounted on a hexagonal base plate using Neewer camera $15 \mathrm{~mm}$ rail systems. Lighting is provided by a top mounted LED ring light, with six additional LEDs mounted in the same plane as the label cameras to reduce label shadows. The lightbox is designed out of OpenBeam components with card side panels and the entire setup is positioned on a Kaiser RS10 copy stand on which the dorsal imaging camera is mounted (Figure 2). 

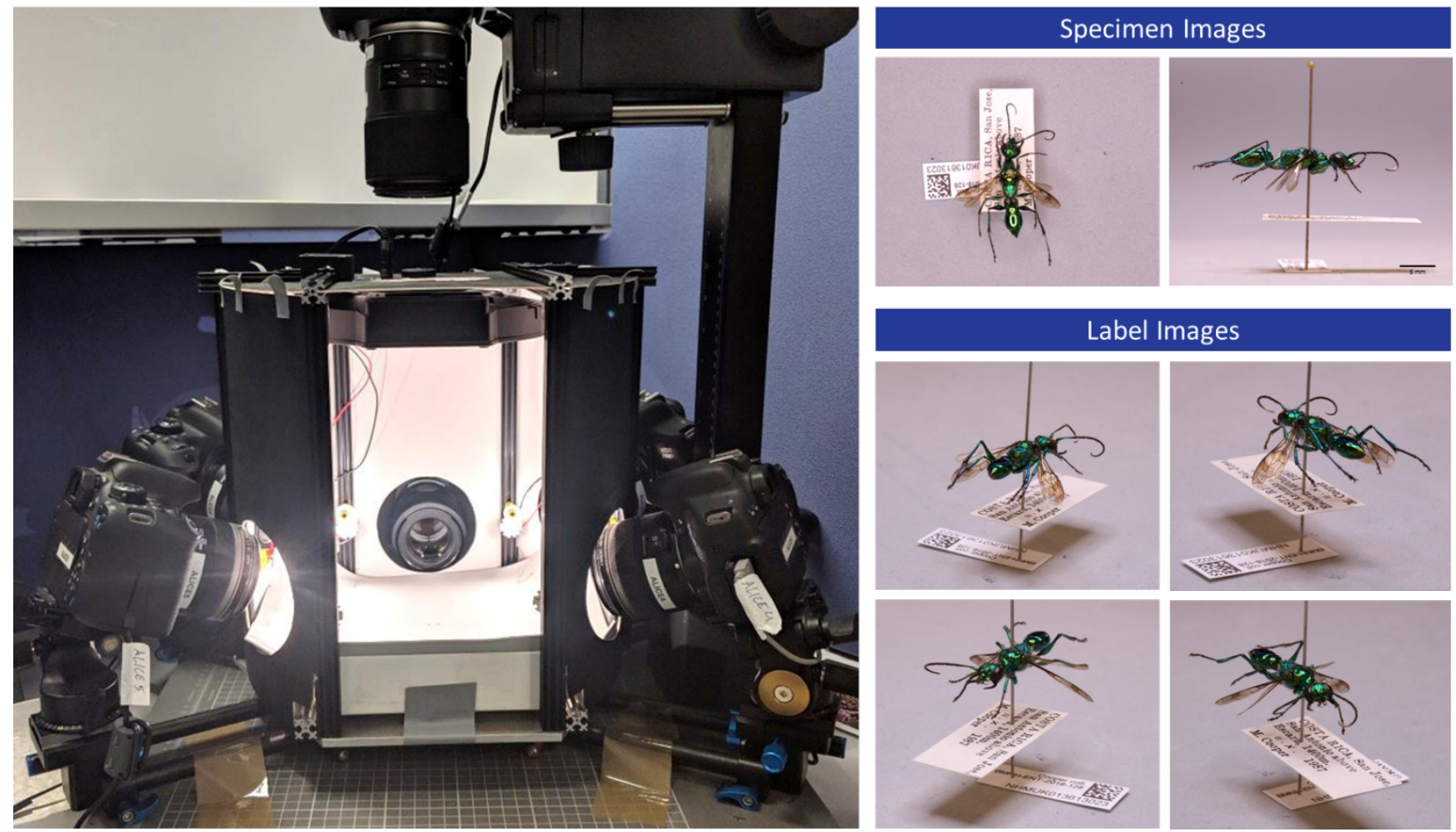

Figure 2: ALICE multi-camera setup in situ and examples of specimen overview and label images captured by each of the cameras. Note: example images are cropped for display.

\section{Camera control}

The cameras are tethered in parallel using DSLR Remote Pro Multi-Camera v.1.9 (Breeze Systems: https://www.breezesys.com/MultiCamera/index.htm). Each camera name is specified using the author token of the image EXIF data (e.g. ALICE1, ALICE2 etc.) and is incorporated into the resulting image filename using the "\%\#\{author\}" command. The camera settings are summarised in Table 1. In addition DigiCamControl v.2.1.0 (http://digicamcontrol.com/) was briefly tested and found to be a suitable free multi-camera tethering alternative.

Table 1: Summary of camera settings

\begin{tabular}{llll}
\hline Camera EXIF name & Make \& Model & View & Image settings \\
\hline ALICE1 & Canon 5DS R & Dorsal & $\mathrm{f} / 20 ; \mathrm{sS}=1 / 80 \mathrm{sec}$; ISO $=400$. \\
ALICE2 & Canon 5DS R & Lateral & $\mathrm{f} / 14 ; \mathrm{ss}=1 / 50 \mathrm{sec} ;$ ISO $=400$. \\
ALICE3; 4; $5 ; 6$ & Canon 750D & Labels & $\mathrm{f} / 20 ; \mathrm{ss}=1 / 50 \mathrm{sec} ;$ ISO $=400$. \\
\hline
\end{tabular}

\section{Camera arrangement}

The top camera ("ALICE1") captures the dorsal view of the specimen, the back camera ("ALICE2") provides a lateral view of the specimen with a scale bar, while the remaining four cameras ("ALICE3; $4 ; 5 ; 6 ")$, which are positioned at $45^{\circ}, 135^{\circ}, 225^{\circ}$ and $315^{\circ}$ to the anterior of the specimen, are directed at the labels at approximately $25^{\circ}$ off the horizontal.

\section{Specimen imaging}

Before specimen imaging begins, a calibration grid (Figure 3 ) is imaged to facilitate automated label transformation. The calibration square is imaged at the start of each imaging session to enable recalibration if the setup has been inadvertently moved. Specimens are labelled with a unique identifier "UID" catalogue number encoded as a Data Matrix barcode and then placed in a predefined central point, designated when the cameras were initially aligned. If required, labels are separated to 
ensure top label data is visible. Temporary labels with the specimen's location in the collection and taxon information, which are linked using database primary key values (i.e. EMu Internal Record Numbers or IRNs) and encoded in a Data Matrix barcode, are also placed in the template in the top left hand corner. These labels are included in the dorsal image and enable automated file renaming in order to integrate with the current NHM mass digitisation workflow (Allan et al. in prep).
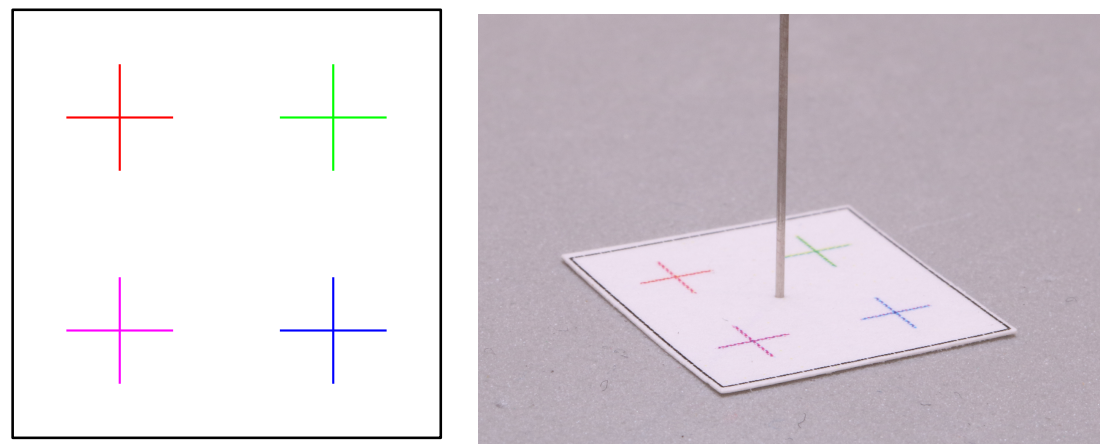

Figure 3: Calibration pattern with example image from ALICE setup, cropped for display. Each cross is separated by $8 \mathrm{~mm}$ (horizontal and vertical, not diagonal distance).

\section{Image processing workflow}

The imaging and file processing workflow (Figure 4) is summarised below.

\section{i) File naming}

Each specimen results in six images, where the first four numbers of the filename are sequential and provide a temporary identifier for each specimen, while the last number corresponds to the camera that has taken the image, encoded by the camera EXIF name (e.g. "0001_ALICE1.jpg"). In order to rename the files to fit the current NHM mass digitisation workflow, the dorsal images are run sequentially through BardecodeFiler v.2.4.4.1 (http://www.bardecode.com/en1/app/bardecodefiler/) and renamed to include the UID as well as the location and taxon primary key values (e.g. "UID_locationIRN_taxonIRN.jpg"). The remaining lateral and label images are then batch renamed using the "rename pairs" function in Bulk Rename Utility v.3.0.0.1

(https://www.bulkrenameutility.co.uk/Main Intro.php), with images named "UID_additional.jpg" for the lateral image and "UID_additional_1/2/3/4.jpg" for the label images.

\section{ii) Basic image manipulation}

Once the images are renamed the dorsal images are batch cropped using XnConvert v.1.74 (https://www.xnview.com/en/xnconvert/) to remove the temporary location and taxon labels from the final image. For lateral images, a scale bar is stamped on the images in batch using an Image $1.52 \mathrm{~h}$ macro within Fiji (https://imagej.net/Fiji/Downloads). Scale bars could only be accurately applied to lateral images as all specimens are imaged at the same distance from the lateral camera. The distance between the dorsal camera and specimen changes slightly due to specimen height on the pin, which could lead to a potential scale inaccuracy of $3 \%$. All six images are then ingested into the collection management system using custom scripts. 


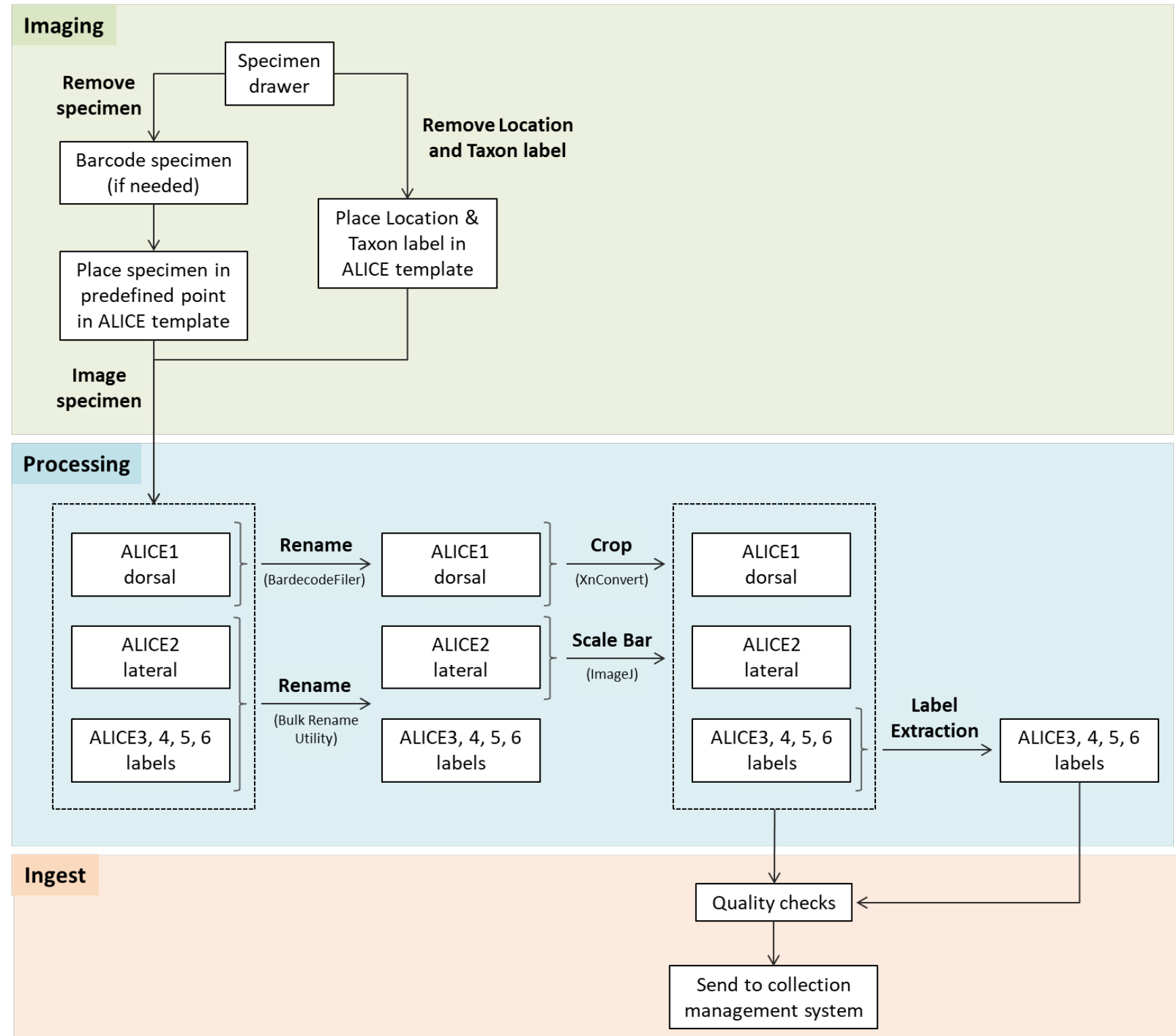

Figure 4: ALICE workflow, including imaging and file processing.

\section{iii) Label detection and extraction}

While the angled images of the labels are legible, and can be used for manual data transcription, they are less amenable to automated text extraction through optical character recognition (OCR) or handwriting recognition software. In order to streamline transcription and facilitate downstream OCR we are developing label extraction software (https://github.com/NaturalHistoryMuseum/ALICE). The label extraction pipeline is currently as follows:

a) Calibration: For each camera angle, image coordinates for a flat calibration square (Figure 3) in world space are calculated by finding the homography (perspective transformation) that aligns all four images. The coordinates are translated to the centre of the image and scaled to fit.

b) Perspective transformation: Each view of the specimen labels is then transformed so that the 'calibration square' would be flat and oriented the same in each image. For anything above this plane (e.g. labels higher up the pin) this step will only approximately align them.

c) Label detection: This is split into three stages:

i) Feature detection: Features can be detected in each image independently and then matched across all perspective corrected images, such that for each detected point we know what its coordinates will be in every other image. 
ii) Feature clustering: Given the correspondences between feature points, they are clustered such that each cluster represents points on the same label.

iii) Cropping: This enables cropping segments from each image to give separate images that each contain the same label and nothing else.

d) Label alignment: Since the perspective correction of the images is only approximate, the cropped label images need to be aligned more closely in order to overlay them correctly. This is done by finding dense correspondences: rather than sparse feature points, this estimates the best correspondence for every pixel. There are likely to be pixels for which this is difficult (for example, if they change appearance due to lighting) or impossible (if a point is not visible in all images), and so it is necessary to also estimate whether these correspondences are reliable or not. A more fine-grained alignment is then computed using only the valid correspondences. For parts of the image where there are no correspondences the alignment will try to keep the image as rigid as possible.

e) Image merging: Once labels are aligned pixel-wise, a median filter is then applied per-pixel so that anything only visible in a minority of views (such as a pin) will no longer be visible.

f) Image processing: Contrast in the merged label is boosted by $5 \%$ for downstream OCR.

g) Result export: Both a single plate of all the resulting labels for a specimen and each identified label are exported for downstream transcription / analysis.

Numerous software packages are currently used in the label extraction pipeline, primarily within the SciPy (https://www.scipy.org) ecosystem: Matplotlib v.3.0.1 for producing figures (Hunter, 2007; Caswell et al., 2018); Numpy v.1.15.0 for numerous mathematical applications; primarily working with image matrices (Oliphant, 2006); SciPy v.1.1.0 for working with sparse matrices (Jones et al., 2001; Olifant 2007; Millman \& Aivazis, 2011); Pandas v.0.23.4 for comparing sets of feature points (McKinney, 2010); scikit-image v.0.14.0 for image analysis and manipulation, e.g. feature detection and matching (van der Walt et al., 2014); scikit-learn v.0.19.2 for clustering feature points (Pedregosa et al., 2011). In addition the pipeline currently uses: cached-property v.1.5.1

(https://github.com/pydanny/cached-property) for caching values that are expensive to calculate; Numba v.0.39.0, which compiles Python functions to machine code for speed improvements (Lam et al., 2015); Pyflow for calculating optical flow between images (Pathak et al., 2017) and pyGCO (https://github.com/Borda/pyGCO) a graph cut optimisation library used here in the feature point clustering stage (Boykov et al., 2001; Boykov \& Kolmogorov, 2004; Kolmogorov \& Zabih, 2004).

\section{Results}

\section{Digitisation rate}

While assessment of the exact timing of each aspect of the digitisation process can help optimise the workflow, we have chosen to report the realistic number of specimens per person per hour, and per person per day, under two typical scenarios in the NHM collections: (1) a collection without UID catalogue numbers added (i.e. process includes labelling and imaging); and (2) a collection with UID catalogue numbers already added (i.e. process is just imaging). Timings were assessed using a three point estimation and the double-triangular distribution technique as in Blagoderov et al. (2017) for comparison. The base rate $(E)$ was calculated using hourly rates from three digitisers to provide a realistic estimate of the rate of imaging for further resource planning (Table 2). Transcription of label data and georeferencing have been assessed elsewhere (Blagoderov et al., 2017) and as these are independent of the imaging workflow they are not included here. 
Table 2: Estimation of the base imaging rate for resource planning.

\begin{tabular}{lrr}
\hline & \multicolumn{3}{c}{ Specimens per person per hour } \\
\cline { 2 - 4 } & $\begin{array}{l}\text { UID catalogue label } \\
\text { added during imaging }\end{array}$ & $\begin{array}{l}\text { UID catalogue label } \\
\text { already added }\end{array}$ \\
\cline { 2 - 3 } $\operatorname{MIN}(\mathrm{a})$ & 96 & 151 \\
$\operatorname{MAX}(\mathrm{b})$ & 188 & 310 \\
$\operatorname{MEDIAN}(\mathrm{m})$ & 139 & 176 \\
BASE $(E)$ & 140 & 194 \\
$E=(a+4 m+b) / 6$ & 15 & 26 \\
Standard Deviation & & \\
SD $=(b-a) / 6$ & & \\
\hline
\end{tabular}

If UID catalogue numbers (encoded as Data Matrix barcodes) are added to specimens during imaging, the base imaging rate $(E)$ is 140 specimens per person per hour, whereas if these labels have already been added during routine curation the imaging rate increases to a base rate of 194 specimens per person per hour (Table 2). Thus in a typical day, which includes 5.5 hours of imaging and 30 minutes of image processing and quality checking, these hourly rates correspond to 770 specimens per person per day including labelling, or 1,067 specimens per person per day when specimens are already labelled. The theoretical maximum rate of image capture using the current multi-camera setup (i.e. without changing specimens) is 1,080 specimens per hour (or 5,940 per setup per day), using a typical desktop PC (Dell Optiplex 3010; CPU: Intel Core i5-3470 @ 3.2 Ghz; RAM: $8 \mathrm{~GB}$; OS: Windows 764 bit). Attempting to capture images faster than this results in image skipping by the dorsal and lateral cameras.

\section{Label analysis}

Analysis of the labels from 700 specimens in seven insect orders (Figure 5) shows that while the number of labels ranged from one to five, the majority ( $80 \%)$ of specimens have two labels or less. Label formats were $46 \%$ printed, $22 \%$ handwritten, and $32 \%$ a mixture of both. 


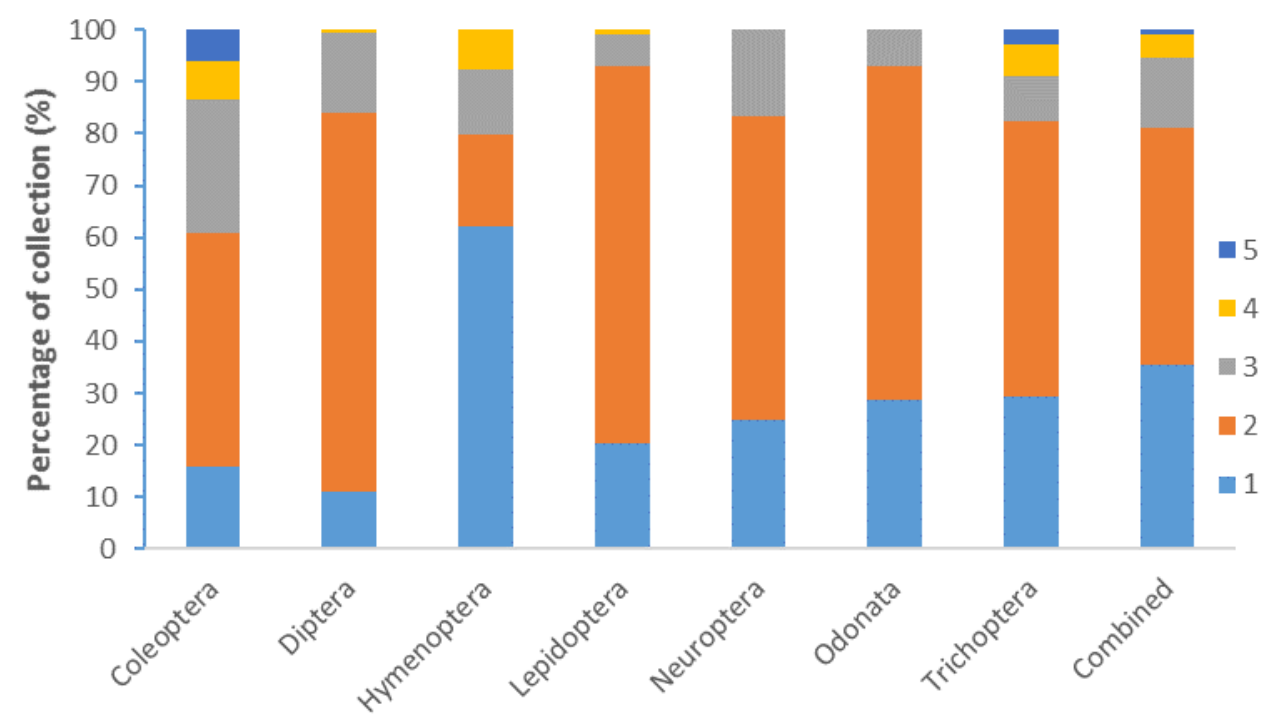

Figure 5: Analysis of the number of labels per specimen from 700 specimens of seven insect orders and all orders combined.

\section{Data capture}

In order to assess the ability of the angled imaging approach to recover primary specimen data, the untransformed label images from 700 specimens were manually assessed for legibility (if present) of: (1) Site; (2) Date collected; and (3) Collector name. This primary data was not assumed as always present as previously highlighted by the iCollections project (Table 3: Blagoderov et al. 2017), rather if data was not visible in the image its presence/absence was confirmed on the physical label.

Analysis of the information content of the angled labels, before transformation, highlighted that collecting locality was almost always visible, being illegible / not visible on $0.1 \%$ specimen images, whereas the collecting date was illegible / not visible on $1.9 \%$ of the specimen images, while collector name was illegible / not visible on $10.0 \%$ of the specimen images.

\section{Label extraction}

Label images were processed using a Dell Precision 7810 PC (CPU: Intel Xeon E5-1650 v3 @ 3.5 GHz [12 cores]; GPU: NVIDIA GeForce GTX 1050; RAM: 64 GB; OS: Ubuntu 16.04). Initial testing took $12 \mathrm{~h} 48 \mathrm{~m} 37 \mathrm{~s}$ to process 772 images, corresponding to 193 specimens that would result in a significant image processing bottleneck. Currently parallelisation of the processing pipeline, either over multiple computers or multiple CPU cores, is being explored to remove this bottleneck. Of the 193 specimens, labels were detected and cropped out in 163 cases $(84.5 \%)$, however this was often only partial label extraction, either only one of multiple labels or parts of multiple labels. In 114 cases $(59.1 \%)$ the label containing the primary data (when, where, who) was extracted from one or more of the angled images, however only 60 specimens (31.1\%) had successful label extraction and merging (e.g. Figure 6). 

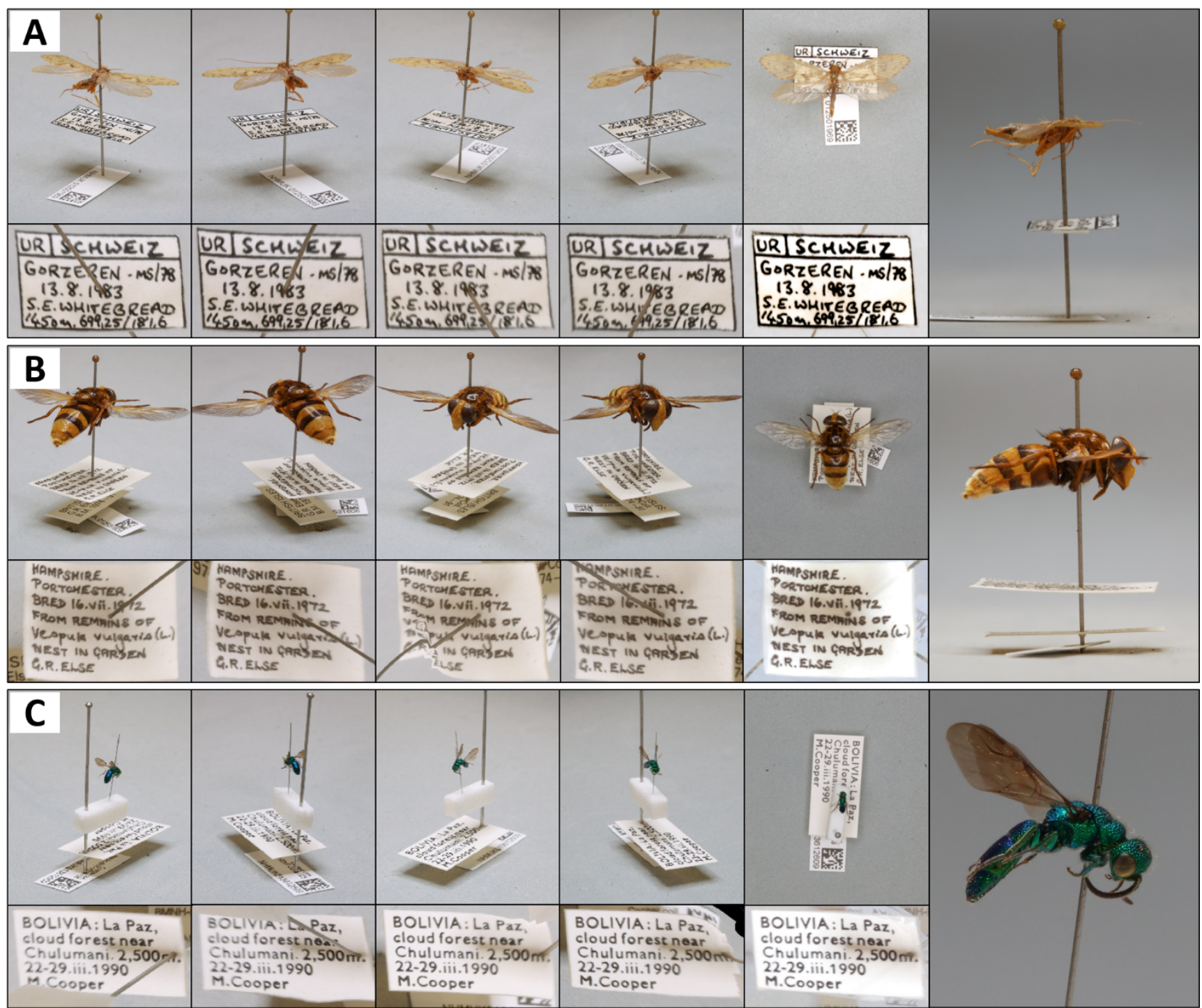

Figure 6: Extracted label from each angled view, with the combined label image under the dorsal view and the lateral image for three successful examples (A) Trichoptera, (B) Diptera, (C) Hymenoptera. Note: Images are cropped from final output for display, in particular the lateral image in (C) is significantly cropped to show the resolution provided by the lateral camera.

\section{Discussion}

The enormous scale of global natural history collections, especially pinned insect collections, requires a major shift in our approach to digitisation in order to unlock the data hidden on their labels in time to apply it to solving the challenges that currently face humanity.

Drawer-level imaging remains the fastest approach to provide an overview of a collection with dorsal specimen images, however it does not offer reliable access to critical label data (Blagoderov et al., 2012; Dietrich et al., 2012; Hudson et al., 2015). Single-specimen imaging with labels removed from the pin remains the most comprehensive approach, capturing all label data 'as is' for reference or transcription, as well as an uninterrupted specimen image, however this approach is slow and the benefits should be weighed against the significant costs before applying this method across all insect groups.

The current six-camera iteration of the ALICE system represents a significant improvement in imaging rates for pinned insect collections at the single specimen level up to $7 x$ the per person rate of the 
NHM iCollections project and $3 x$ the fastest published per person rate using a similar angled approach (Tegelberg et al., 2017). This multi-camera approach allows a single user to capture dorsal and lateral specimen images, along with images of key label data at a realistic rate of 1,067 specimens per person per day. The label data is captured in a way that can subsequently be transcribed, either manually or using automated approaches such as OCR and handwriting recognition following label transformation and merger. At this rate creating a specimen inventory with associated label images of the NHM pinned insect collection would take 106 person years. Manual data transcription will take another 173 person years using the iCollections estimate (Blagoderov et al., 2017), thus it is clear that the data extraction bottleneck requires further exploration of OCR and handwriting recognition.

The maximum imaging rate achievable using ALICE is 1,080 specimens per hour; while a conveyor feed system with multiple operators could theoretically reach this imaging rate, the per person rate would not likely improve due to the bottleneck of safe specimen handling. Given the additional specimen movement that could result in increased damage, the design complexity and the significant space requirements for such a conveyor system we have opted to focus for now on a compact, single user, desk based setup, using commercially available components and minimal bespoke elements.

It is clear that the ALICE approach is not suitable for all pinned insect collections, for example large specimens that completely cover the labels (e.g. "macrolepidoptera") or where labels are reversed are unlikely to be amenable to this current approach. We are exploring the use of mirrors in the setup to overcome the issue of reversed labels (e.g. Tegelberg et al., 2014) and additional cameras at different orientations and a shallower angle (ie. less than $25 \%$ ) could potentially recover information from closely spaced labels or larger specimens. Specimens where the labels are pushed together will need separation before imaging if the primary data is not visible, reducing rates slightly, though this was only required in a very small minority of cases during our limited testing. With these caveats in mind and when applied to amenable collections (e.g. Diptera, Hymenoptera, Hemiptera, Coleoptera) the current six-camera approach is capable of recovering primary specimen data in the vast majority (over 90\%) of cases from the untransformed label images. Furthermore, the addition of two specimen views (dorsal and lateral), while not necessarily diagnostic, are able to give an overview of each specimen for external users, including relatively small specimens (e.g. wasp specimen: Figure 6C).

The current label extraction and merging software pipeline is not efficient, taking several minutes per specimen, but processing efficiency is not the primary focus of the research at this time as compute resources are relatively inexpensive and we are currently investigating parallelisation to increase processing efficiency. The pipeline is not particularly robust at present, recovering only $59 \%$ of the primary data labels from the initial test specimens, with only $31 \%$ being successfully merged into a single image for downstream manual transcription or automated OCR / handwriting recognition. The current approach assumes that each label is flat, which makes feature matching more difficult when they are even slightly curved, thus we are exploring methods to estimate and rectify the curvature before merging different views of each label and are confident that we can increase the label extraction and merger success rate in the near future.

In conclusion, the multi-camera angled approach to label imaging, combined with downstream label extraction, promises to substantially improve the rate of specimen digitisation of pinned insects. While this is only one approach to accelerate data capture with specimen and label imaging we hope that the hardware and software outlined here will assist in unlocking this unique resource: the data held on hundreds of millions of pins in global natural history collections. 


\section{Data availability}

The label extraction software is available from: https://github.com/NaturalHistoryMuseum/ALICE. The images used for software development and to assess future label extraction pipelines can be downloaded from the Natural History Museum Data Portal: https://doi.org/10.5519/0072507.

\section{Acknowledgements}

We thank Scott Fynn for helpful discussion around label transformation; Beulah Garner for testing an early version of the imaging system; Daniel Whitmore, Nigel Wyatt and David Lees for providing test specimens and Anthony Roach for early testing of the rotational stage. This research received support from the European Union's Horizon 2020 research and innovation programme under grant agreement No 777483 "ICEDIG"; the SYNTHESYS Project, http://www.synthesys.info/, which is financed by European Community Research Infrastructure Action under the FP7 Integrating Activities Programme (Grant agreement number 312253); and the Natural History Museum's Digital Collections Programme.

\section{References}

Allan EL, Livermore L, Price BW, Shchedrina O, Smith VS (in prep) A Novel Automated Mass Digitisation Workflow for Natural History Microscope Slides. Biodiversity Data Journal.

Ariño $\mathrm{AH}$ (2010) Approaches to estimating the universe of natural history collections data. Biodiversity Informatics 7: 81-92. https://doi.org/10.17161/bi.v7i2.3991

Blagoderov V, Kitching I, Livermore L, Simonsen T, Smith V (2012) No specimen left behind: industrial scale digitization of natural history collections. ZooKeys 209: 133-146. https://doi.org/10.3897/zookeys.209.3178

Blagoderov V, Penn M, Sadka M, Hine A, Brooks S, Siebert D, Sleep C, Cafferty S, Cane E, Martin G, Toloni F, Wing P, Chainey J, Duffell L, Huxley R, Ledger S, McLaughlin C, Mazzetta G, Perera J, Crowther R, Douglas L, Durant J, Scialabba E, Honey M, Huertas B, Howard T, Carter V, Albuquerque S, Paterson G, Kitching I (2017) iCollections methodology: workflow, results and lessons learned. Biodiversity Data Journal 5: e21277. https://doi.org/10.3897/BDJ.5.e21277

Boykov Y, Kolmogorov V (2004) An Experimental Comparison of Min-Cut/Max-Flow Algorithms for Energy Minimization in Vision. IEEE TPAMI 26(9): 1124-1137.

Boykov Y, Veksler O, Zabih R (2001) Efficient Approximate Energy Minimization via Graph Cuts. IEEE TPAMI 20(12): 1222-1239.

Caswell TA, Droettboom M, Hunter J, Firing E, Lee A, Nielsen JH et al. (2018) matplotlib/matplotlib v3.0.0. Zenodo http://doi.org/10.5281/zenodo.1420605

Dietrich C, Hart J, Raila D, Ravaioli U, Sobh N, Sobh O, Taylor C (2012) InvertNet: a new paradigm for digital access to invertebrate collections. ZooKeys 209: 165-181. https://doi.org/10.3897/zookeys.209.3571

Hereld M, Ferrier NJ, Agarwal N, Sierwald P (2017) Designing a High-Throughput Pipeline for Digitizing Pinned Insects. IEEE 13th International Conference on e-Science (e-Science), Auckland, pp. 542-550. https://doi.org/10.1109/eScience.2017.88

Hudson LN, Blagoderov V, Heaton A, Holtzhausen P, Livermore L, Price BW, van der Walt S, Smith VS (2015) Inselect: Automating the Digitization of Natural History Collections. PLOS ONE 10(11): e0143402. https://doi.org/10.1371/journal.pone.0143402

Hunter JD (2007) Matplotlib: A 2D Graphics Environment. Computing in Science \& Engineering 9: 9095. https://doi.org/10.1109/MCSE.2007.55 
Jones E, Oliphant TE, Peterson P, et al. (2001) SciPy: Open Source Scientific Tools for Python, 2001, http://www.scipy.org/ [Online; accessed 2018-11-03].

Kolmogorov V, Zabih R (2004) What Energy Functions can be Minimized via Graph Cuts? IEEE TPAMI, 26(2): 147-159.

Lam SK, Pitrou A, Seibert S (2015) Numba: a LLVM-based Python JIT compiler. In Proceedings of the Second Workshop on the LLVM Compiler Infrastructure in HPC (LLVM '15). ACM, New York, NY, USA, Article 7, 6 pages. https://doi.org/10.1145/2833157.2833162

Lewin HA, Robinson GE, Kress WJ, Baker WJ, Coddington J, Crandall KA, Durbin R, Edwards SV, Forest F, Gilbert MTP, Goldstein MM, Grigoriev IV, Hackett KJ, Haussler D, Jarvis ED, Johnson WE, Patrinos A, Richards S, Castilla-Rubio JC, Sluys MA, Soltis PS, Xu X, Yang $H$, Zhang G (2018) Earth BioGenome Project: Sequencing life for the future of life. Proceedings of the National Academy of Sciences 115(17): 4325-4333.

https://doi.org/10.1073/pnas.1720115115

McKinney W (2010) Data Structures for Statistical Computing in Python, Proceedings of the 9th Python in Science Conference, 51-56. van der Walt S and Millman J (Eds) ISBN-13: 9781-4583-4619-3.

Millman KJ, Aivazis M (2011) Python for Scientists and Engineers. Computing in Science \& Engineering 13: 9-12. https://doi.org/10.1109/MCSE.2011.36

Nguyen C, Adcock M, Anderson S, Lovell D, Fisher N, La Salle J (2017) Towards High-Throughput 3D Insect Capture for Species Discovery and Diagnostics. IEEE 13th International Conference on e-Science (e-Science), Auckland, 2017, pp. 559-560.

https://doi.org/10.1109/eScience.2017.90

Oliphant TE (2006) A guide to NumPy. Trelgol Publishing, USA, 85pp.

Oliphant TE (2007) Python for Scientific Computing. Computing in Science \& Engineering 9: 10-20. https://doi.org/10.1109/MCSE.2007.58

Paterson G, Albuquerque S, Blagoderov V, Brooks S, Cafferty S, Cane E, Carter V, Chainey J, Crowther R, Douglas L, Durant J, Duffell L, Hine A, Honey M, Huertas B, Howard T, Huxley R, Kitching I, Ledger S, McLaughlin C, Martin G, Mazzetta G, Penn M, Perera J, Sadka M, Scialabba E, Self A, Siebert D, Sleep C, Toloni F, Wing P (2016) iCollections Digitising the British and Irish Butterflies in the Natural History Museum, London.

Biodiversity Data Journal 4: e9559. https://doi.org/10.3897/BDJ.4.e9559

Pathak D, Girshick R, Dollár P, Darrell T Hariharan B (2017) Learning Features by Watching Objects Move. CVPR 2017. https://arxiv.org/abs/1612.06370

Pedregosa F, Varoquaux G, Gramfort A, Michel V, Thirion B, Grisel O, Blondel M, Prettenhofer P, Weiss R, Dubourg V, Vanderplas J, Passos A, Cournapeau D, Brucher M, Perrot M, Duchesnay E (2011) Scikit-learn: Machine Learning in Python Journal of Machine Learning Research 12: 2825-2830.

Suarez AV, Tsutsui ND (2004) The Value of Museum Collections for Research and Society, BioScience 54(1): 66-74. https://doi.org/10.1641/0006-

3568(2004)054[0066:TVOMCF]2.0.CO;2

Tegelberg R, Kahanpää J, Karppinen J, Mononen T, Wu Z, Saarenmaa H (2017) Mass Digitization of Individual Pinned Insects Using Conveyor-Driven Imaging. IEEE 13th International Conference on e-Science (e-Science), Auckland, 2017, pp. 523-527. https://doi.org/10.1109/eScience.2017.85

Tegelberg R, Mononen T, Saarenmaa, H (2014) High-performance digitization of natural history collections: Automated imaging lines for herbarium and insect specimens. Taxon 63:13071313. https://doi.org/10.12705/636.13

van der Walt S, Schönberger JL, Nunez-Iglesias J, Boulogne F, Warner JD, Yager N, Gouillart E, Yu T and the scikit-image contributors (2014) scikit-image: Image processing in Python, PeerJ 2:e453. https://doi.org/10.7717/peeri.453 